\title{
Simplified Machine Diagnosis Techniques with Absolute Deterioration Factor by Utilizing the Second Order Autocorrelation Function
}

\author{
Kazuhiro Takeyasu ${ }^{1}$ \\ ${ }^{1}$ College of Business Administration, Tokoha University, Japan \\ Correspondence: Kazuhiro Takeyasu, College of Business Administration, Tokoha University, Japan
}

Received: December 5, 2018

Accepted: January 4, 2019

Online Published: January 8, 2019

doi:10.5430/bmr.v8n1p1

URL: https://doi.org/10.5430/bmr.v8n1p1

\begin{abstract}
Among many amplitude parameters, Kurtosis and Bicoherence are recognized to be the sensitive good parameters for machine diagnosis. In this paper, simplified calculation method of coefficient of autocorrelation function of $1^{\text {st }}$ order lag is introduced, and new deterioration factor of correlation of four variables is introduced consecutively. New deterioration factor is an absolute index of deterioration factor. Three cases in which the rolling elements number is nine, twelve and sixteen are examined and compared. Varying N, the number of data, its characteristics are examined. When $\mathrm{N}$ is smaller, sensitivity is better. Therefore this parameter can be used as an index for the machine diagnosis of early failure stage especially.
\end{abstract}

Keywords: impact vibration, deterioration, autocorrelation function, rolling element

\section{Introduction}

In mass production firms such as steel making that have big equipments, sudden stops of production processes by machine failure cause great damage such as shortage of materials to the later processes, delays to the due date and increased idling time.

To prevent these troubles, machine diagnosis techniques play important roles. So far, Time Based Maintenance (TBM) technique has constituted the main stream of the machine maintenance, which makes checks for maintenance at previously fixed times. But it has a weak point in that it makes checks at scheduled times without taking into account whether the parts are still in good condition or not. On the other hand, Condition Based Maintenance (CBM) makes maintenance checks by watching the condition of machines. Therefore, if the parts are still keeping good condition beyond its supposed life, the cost of maintenance may be saved because machines can be used longer than planned. Therefore the use of CBM has become dominant. The latter one needs less cost of parts, less cost of maintenance and leads to lower failure ratio.

However, it is mandatory to catch a symptom of the failure as soon as possible if a transition from TBM to CBM is to be made. Many methods are developed and examined focusing on this subject. In this paper, we propose a method for the early detection of the failure on rotating machines that is the most common theme in machine failure detection field.

So far, many signal processing methods for machine diagnosis have been proposed (Bolleter, 1998). As for sensitive parameters, Kurtosis, Bicoherence, Impact Deterioration Factor (ID Factor) were examined (Yamazaki, 1977; Maekawa et al.,1997; Shao et al.,2001; Song et al.,1998; Takeyasu,1989). In this paper, we focus our attention to the index parameters of vibration.

Kurtosis is one of the sophisticated inspection parameters that calculates normalized $4^{\text {th }}$ moment of Probability Density Function (PDF). In the industry, there are cases where quick reactions are required on watching the waveform at the machine site.

In this paper, we consider the case such that the impact vibration occurs on the gear when the failure arises.

In this paper, we introduce a new absolute deterioration factor of correlation of four variables and examine that it is a sensitive good parameter for machine diagnosis. Three cases in which the rolling elements number is nine, twelve and sixteen are examined and compared. This method is simple enough to calculate even on a pocketsize calculator and enables us to install it in microcomputer chips. 
The rest of the paper is organized as follows. We survey each index of deterioration in section 2. Simplified calculation method of autocorrelation function is introduced in section 3. New absolute deterioration factor of correlation of four variables is introduced in section 4 , and numerical example is exhibited in section 5 followed by Remarks of section 6. Section 7 is a summary.

\section{Factors for Vibration Calculation}

In cyclic movements such as those of bearings and gears, the vibration grows larger whenever the deterioration becomes bigger. Also, it is well known that the vibration grows large when the setting equipment to the ground is unsuitable (Yamazaki, 1997). Assume the vibration signal is the function of time as $x(t)$. And also assume that it is a stationary time series with mean 0 . Denote the probability density function of these time series as $p(x)$. Indices for vibration amplitude are as follows.

$$
\begin{aligned}
& X_{\text {root }}=\left[\int_{-\infty}^{\infty}|x|^{\frac{1}{2}} p(x) d x\right]^{2} \\
& X_{r m s}=\left[\int_{-\infty}^{\infty} x^{2} p(x) d x\right]^{\frac{1}{2}} \\
& X_{a b s}=\int_{-\infty}^{\infty}|x| p(x) d x \\
& X_{\text {peak }}=\lim _{n \rightarrow \infty}\left[\int_{-\infty}^{\infty} x^{n} p(x) d x\right]^{\frac{1}{n}}
\end{aligned}
$$

These are dimensional indices that are not normalized. They differ by machine sizes or rotation frequencies. Therefore, normalized dimensionless indices are required.
A. Normalized root mean square value
B. Normalized peak value
C. Normalized moment
D. Normalized correlation among frequency domain

A. Normalized root mean square value

a. Shape Factor: SF

$$
S F=\frac{X_{r m s}}{\bar{X}_{a b s}}
$$

( $\bar{X}_{a b s}$ : mean of the absolute value of vibration)

B. Normalized peak value

b. Crest Factor: $\mathrm{CrF}$

$$
C r F=\frac{X_{p e a k}}{X_{r m s}}
$$

( $X_{\text {peak }}$ : peak value of vibration)

c. Clearance Factor: $\mathrm{ClF}$

$$
C l F=\frac{X_{\text {peak }}}{X_{\text {root }}}
$$

d. Impulse Factor: IF

$$
I F=\frac{X_{p e a k}}{\bar{X}_{a b s}}
$$

e. Impact Deterioration Factor: ID Factor

$$
I D=\frac{X_{p e a k}}{X_{c}}
$$


( $X_{c}$ : vibration amplitude where the curvature of PDF becomes maximum)

C. Normalized moment

f. Skewness: SK

$$
S K=\frac{\int_{-\infty}^{\infty} x^{3} p(x) d x}{\left[\int_{-\infty}^{\infty} x^{2} p(x) d x\right]^{\frac{3}{2}}}
$$

g. Kurtosis: KT

$$
K T=\frac{\int_{-\infty}^{\infty} x^{4} p(x) d x}{\left[\int_{-\infty}^{\infty} x^{2} p(x) d x\right]^{2}}
$$

D. Normalized correlation among frequency domain

h. Bicoherence

Bicoherence means the relationship of a function at different points in the frequency domain and is expressed as:

$$
\text { Bic },_{x x x}\left(f_{1}, f_{2}\right)=\frac{B_{x x x}\left(f_{1}, f_{2}\right)}{\sqrt{S_{x x}\left(f_{1}\right) \cdot S_{x x}\left(f_{2}\right) \cdot S_{x x}\left(f_{1}+f_{2}\right)}}
$$

Here

$$
B_{x x x}\left(f_{1}, f_{2}\right)=\frac{X_{T}\left(f_{1}\right) \cdot X_{T}\left(f_{2}\right) \cdot X_{T}^{*}\left(f_{1}+f_{2}\right)}{T^{\frac{3}{2}}}
$$

means Bispectrum and

$$
X_{T}(t)= \begin{cases}x(t) & (0<t<T) \\ 0 & (\text { else })\end{cases}
$$

$\mathrm{T}:$ Basic Frequency Interval

$$
\begin{aligned}
& X_{T}(t)=\int_{-\infty}^{\infty} X_{T}(t) e^{-j 2 \pi f t} d t \\
& S_{x x}(f)=\frac{1}{T} X_{T}(f) X_{T}^{*}(f)
\end{aligned}
$$

Range of Bicoherence satisfies

$$
0<\text { Bic },_{x x x}\left(f_{1}, f_{2}\right)<1
$$

When these exists a significant relationship between frequencies $f_{1}$ and $f_{2}$, Bicoherence is near 1 and otherwise comes close to 0 .

These indices are generally used in combination and machine condition is judged totally. Among them, Kurtosis is recognized to be the superior index (Noda, 1987) and many researches on this have been made (Maekawa et al., 1997; Shao et al., 2001; Song et al., 1998). Judging from the experiment we made in the past, we may conclude that Bicoherence is also a sensitive good index (Takeyasu, 1989). In Maekawa et al. (1997), ID Factor is proposed as a good index. In this paper, we focus on the indices of vibration amplitude, and we introduce the deterioration factor of correlation of four variables.

\section{Simplified Calculation Method of Deterioration Factor of Autocorelation Function Type}

In discrete time series, mean of $\left\{x_{i}\right\}$ is stated as follows.

$$
\bar{x}=\frac{1}{N} \sum_{i=1}^{N} x_{i}
$$


Variance of $\left\{x_{i}\right\}$ is stated as follows.

$$
\sigma^{2}=\frac{1}{N} \sum_{i=1}^{N}\left(x_{i}-\bar{x}\right)^{2}
$$

Autocorrelation of $\left\{x_{i}\right\}$ is defined as follows.

$$
\begin{gathered}
R_{l}=E\left[x_{i} x_{i+l}\right]=\frac{1}{N-l} \sum_{i=1}^{N-l} x_{i} x_{i+l} \\
(l=0,1,2, \cdots)
\end{gathered}
$$

As $\left\{x_{i}\right\}$ is assumed to be stationary time series, we can assume $E\left[x_{i}\right]=0$ without loss of generality (Tokumaru et al., 1982). Therefore, (17) can be re-stated as:

$$
\begin{aligned}
R_{l} & =E\left[\left(x_{i}-\bar{x}\right)\left(x_{i+l}-\bar{x}\right)\right] \\
& =\frac{1}{N-l} \sum_{i=1}^{N-l}\left(x_{i}-\bar{x}\right)\left(x_{i+l}-\bar{x}\right)
\end{aligned}
$$

That is to say, $0^{\text {th }}$ order autocorrelation function is a variance itself.

Coefficient of autocorrelation function is stated as :

$$
\rho_{l}=\frac{R_{l}}{R_{0}}
$$

Assume that the peak signal which has $S$ times impact from normal signal arises in each $m$ times samplings. As for determining the sampling interval, the sampling theorem, which is well known, can be used (Tokumaru et al., 1982). But in this paper, we do not pay much attention on this point in order to focus on our proposal theme.

Let of this case be $\bar{\sigma}^{2}$, then we get :

$$
\begin{aligned}
\bar{\sigma}^{2} & =\frac{1}{N} \sum_{i=1}^{N}\left(x_{i}-\bar{x}\right)^{2} \\
& \cong \frac{N-\frac{N}{m}}{N} \sigma^{2}+\frac{N}{N} S^{2} \sigma^{2} \\
& =\left(1+\frac{S^{2}-1}{m}\right) \sigma^{2}
\end{aligned}
$$

For about autocorrelation function, let of this case be $\bar{R}_{i} \quad(i=0,1,2, \cdots)$ in the same way. $0^{\text {th }}$ autocorrelation function is variance, so we can get:

$$
\bar{R}_{0}=\bar{\sigma}^{2} \cong\left(1+\frac{S^{2}-1}{m}\right) \sigma^{2}
$$

In the next, we examine the feature of autocorrelation function of $1^{\text {st }}$ order lag. From definition, $\bar{R}_{1}$ is stated as 
follows.

$$
\bar{R}_{1}=\frac{1}{N-1} \sum_{i=1}^{N-1} x_{i} x_{i+1}
$$

When the peak signal of $S$ times impact from normal signal arises in each $m$ times samplings, following ^ parts of $\left\{x_{i}\right\}$ may be considered to have peak value during the calculation of $R_{1}$.

$$
1,2, \cdots, m-2, m-1, m, m+1, m+2, \cdots, 2 m-1,2 m, 2 m+1, \cdots
$$

\section{$\wedge \wedge \wedge \wedge$}

From these, there arise following products.

1 product with peak value, $\{(m-1)-1\}$ products with ordinary level up to $m$

3 products with peak value, $\{(2 m-1)-3\}$ products with ordinary level up to $2 m$

$(2 k-1)$ products with peak value, $\{(k m-1)-(2 k-1)\}$ products with ordinary level up to $\mathrm{km}$

$2 k$ products with peak value, $\{(k m+\alpha-1)-2 k\}$ products with ordinary level up to $k m+\alpha$

Therefore, when $N=k m$ (case 1 )

$\left(2 \frac{N}{m}-1\right)$ products with peak value, $\left\{(N-1)-\left(2 \frac{N}{m}-1\right)\right\}$ products with ordinary level up to $N$

Or, when $N=k m+\alpha(1 \leq \alpha \leq m-1)$ (case 2)

$\left(2 \frac{N-\alpha}{m}\right)$ products with peak value, $\left\{(N-1)-\left(2 \frac{N-\alpha}{m}\right)\right\}$ products with ordinary level up to $N$

When $S$ is large, we make a simplified calculation.

Suppose

$$
\begin{gathered}
\bar{x}-\varepsilon<x_{i}<\bar{x}+\varepsilon \\
\varepsilon>0,(i=1,2, \cdots, N)
\end{gathered}
$$

Then, we get the following relations.

$<$ Case 1>

When $\bar{x}>0$

$\frac{1}{N-1}\left\{S\left(2 \frac{N}{m}-1\right)+(N-1)-\left(2 \frac{N}{m}-1\right)\right\}(\bar{x}-\varepsilon)^{2}<R_{1}<\frac{1}{N-1}\left\{S\left(2 \frac{N}{m}-1\right)+(N-1)-\left(2 \frac{N}{m}-1\right)\right\}(\bar{x}+\varepsilon)^{2}$

When $\bar{x}<0$

$\frac{1}{N-1}\left\{S\left(2 \frac{N}{m}-1\right)+(N-1)-\left(2 \frac{N}{m}-1\right)\right\}(\bar{x}-\varepsilon)^{2}>R_{1}>\frac{1}{N-1}\left\{S\left(2 \frac{N}{m}-1\right)+(N-1)-\left(2 \frac{N}{m}-1\right)\right\}(\bar{x}+\varepsilon)^{2}$ 
When $\bar{x} \rightarrow 0$ and $\sigma^{2} \cong \varepsilon^{2}$, we get the following equation from (21).

$$
\begin{gathered}
\bar{\rho}_{1}=\frac{\bar{R}_{1}}{\bar{R}_{0}} \\
\cong \frac{1}{N-1}\left[S\left(2 \frac{N}{m}-1\right)+(N-1)-\left(2 \frac{N}{m}-1\right)\right] \cdot \frac{1}{1+\frac{S^{2}-1}{m}} \\
=\frac{S(2 N-m)+(m-2) N}{(N-1)\left(m+S^{2}-1\right)}
\end{gathered}
$$

$<$ Case 2>

When $\bar{x}>0$

$$
\frac{1}{N-1}\left\{S\left(2 \frac{N-\alpha}{m}\right)+(N-1)-\left(2 \frac{N-\alpha}{m}\right)\right\}(\bar{x}-\varepsilon)^{2}<R_{1}<\frac{1}{N-1}\left\{S\left(2 \frac{N-\alpha}{m}\right)+(N-1)-\left(2 \frac{N-\alpha}{m}\right)\right\}(\bar{x}+\varepsilon)^{2}
$$

When $\bar{x}<0$

$$
\frac{1}{N-1}\left\{S\left(2 \frac{N-\alpha}{m}\right)+(N-1)-\left(2 \frac{N-\alpha}{m}\right)\right\}(\bar{x}-\varepsilon)^{2}>R_{1}>\frac{1}{N-1}\left\{S\left(2 \frac{N-\alpha}{m}\right)+(N-1)-\left(2 \frac{N-\alpha}{m}\right)\right\}(\bar{x}+\varepsilon)^{2}
$$

When $\bar{x} \rightarrow 0$ and $\sigma^{2} \cong \varepsilon^{2}$, we get the following equation from (21).

$$
\begin{gathered}
\bar{\rho}_{1}=\frac{\bar{R}_{1}}{\bar{R}_{0}} \\
\cong \frac{1}{N-1}\left[S\left(2 \frac{N-\alpha}{m}\right)+(N-1)-\left(2 \frac{N-\alpha}{m}\right)\right] \cdot \frac{1}{1+\frac{S^{2}-1}{m}} \\
=\frac{2(S-1)(N-\alpha)+m(N-1)}{(N-1)\left(m+S^{2}-1\right)}
\end{gathered}
$$

When $\alpha=1$, it is a singular case where the value becomes same for any $N$.

(25) and (28) are the simplified calculation of coefficient of autocorrelation function of $1^{\text {st }}$ order lag. Under normal conditions, $\bar{\rho}_{1}=1$. When $N \rightarrow \infty, S \rightarrow \infty, \bar{\rho}_{1} \rightarrow 0$. This deterioration factor has the characteristics that when the system is under normal condition, it is 1 or close to 1 , and when abnormal condition proceeds, it tends to 0 .

\section{Second Order Autocorrelation Function}

Using the following relation (Nakamizo, 1988),

$$
E[a b c d]=E[a b] E[c d]+E[a c] E[b d]+E[a d] E[b c]
$$

when variables are $x(t), x\left(t+\tau_{1}\right), x\left(t+\tau_{2}\right), x\left(t+\tau_{3}\right)$, 
we get the following equation.

$$
\begin{aligned}
& E\left[x(t) x\left(t+\tau_{1}\right) x\left(t+\tau_{2}\right) x\left(t+\tau_{3}\right)\right] \\
= & R\left(\tau_{1}\right) R\left(\tau_{3}-\tau_{2}\right)+R\left(\tau_{2}\right) R\left(\tau_{3}-\tau_{1}\right)+R\left(\tau_{3}\right) R\left(\tau_{2}-\tau_{1}\right)
\end{aligned}
$$

Set $\tau_{3}=\tau_{1}, \tau_{2}=0$. Then we get the following equation.

$$
\begin{aligned}
& E\left[x(t)^{2} x\left(t+\tau_{1}\right)^{2}\right] \\
= & R(0)^{2}+2 R\left(\tau_{1}\right)^{2}
\end{aligned}
$$

In other words, this is said to be a second order correlation.

Here, we introduce the following number.

$$
J K=\frac{1+2\left(\frac{R\left(\tau_{1}\right)}{R(0)}\right)^{2}}{3}
$$

Set $J K$ as $\overline{J K}$, in the case that the peak signal which has $S$ times impact from normal signal arises in each $m$ times samplings. Then we get the following equation

$$
\overline{J K}=\frac{1+2 \bar{\rho}_{1}^{2}}{3}
$$

The absolute deterioration factor such as Bicoherence is easy to handle because it takes the value of 1.0 under the normal condition and tends to be 0 when damages increase. Therefore we introduce the following equation, which would make an absolute deterioration factor.

$$
Z=\frac{3}{2}\left(\overline{J K}-\frac{1}{3}\right)
$$

Under the normal condition, $Z$ is 1 and tends to be 0 when damages increase.

Therefore, $\bar{Z}=1$ when $S=1$. When $N \rightarrow \infty$ and $S \rightarrow \infty, \bar{Z} \rightarrow 0$.

\section{Numerical Example}

Under the assumption of 3 , let $m=9,12,16$. Here, $m$ is the number of rolling elements. Considering the case

$S=2,4,6,8$ and the case $N=100,1000,10000$, we obtain Table1 from the calculation of (34).

Table1. $Z$ by the variation of $S$

$<m=9$ >

$$
N=100 \quad(\alpha=1)
$$

\begin{tabular}{|c|ccccc|}
\hline$S$ & 1 & 2 & 4 & 6 & 8 \\
\hline$Z$ & 1.0 & 0.84028 & 0.39063 & 0.18647 & 0.10204 \\
\hline
\end{tabular}

$$
N=1000 \quad(\alpha=1)
$$

\begin{tabular}{|c|ccccc|}
\hline$S$ & 1 & 2 & 4 & 6 & 8 \\
\hline$Z$ & 1.0 & 0.84028 & 0.39063 & 0.18647 & 0.10204 \\
\hline
\end{tabular}


$N=10000(\alpha=1)$

\begin{tabular}{|c|ccccc|}
\hline$S$ & 1 & 2 & 4 & 6 & 8 \\
\hline$Z$ & 1.0 & 0.84028 & 0.39063 & 0.18647 & 0.10204 \\
\hline
\end{tabular}

$\langle m=12>$
$N=100 \quad(\alpha=4)$
\begin{tabular}{|c|ccccc|}
\hline$S$ & 1 & 2 & 4 & 6 & 8 \\
\hline$Z$ & 1.0 & 0.86358 & 0.43551 & 0.21311 & 0.111629 \\
\hline
\end{tabular}

$N=1000 \quad(\alpha=4)$
\begin{tabular}{|c|ccccc|}
\hline$S$ & 1 & 2 & 4 & 6 & 8 \\
\hline$Z$ & 1.0 & 0.87036 & 0.44356 & 0.21801 & 0.11997 \\
\hline
\end{tabular}

$N=10000(\alpha=4)$

\begin{tabular}{|c|ccccc|}
\hline$S$ & 1 & 2 & 4 & 6 & 8 \\
\hline$Z$ & 1.0 & 0.87103 & 0.44436 & 0.21904 & 0.12014 \\
\hline
\end{tabular}

$\langle m=16>$
$N=100 \quad(\alpha=4)$
\begin{tabular}{|c|ccccc|}
\hline$S$ & 1 & 2 & 4 & 6 & 8 \\
\hline$Z$ & 1.0 & 0.89148 & 0.49535 & 0.25387 & 0.14016 \\
\hline
\end{tabular}

$N=1000 \quad(\alpha=8)$
\begin{tabular}{|c|ccccc|}
\hline$S$ & 1 & 2 & 4 & 6 & 8 \\
\hline$Z$ & 1.0 & 0.89611 & 0.50172 & 0.25850 & 0.14327 \\
\hline
\end{tabular}

$N=10000 \quad(\alpha=0)$
\begin{tabular}{|c|ccccc|}
\hline$S$ & 1 & 2 & 4 & 6 & 8 \\
\hline$Z$ & 1.0 & 0.89736 & 0.50345 & 0.25976 & 0.14411 \\
\hline
\end{tabular}

Generally, when $N$ grows large, the value decreases slightly except for the singular case $\alpha=1$. Under the singular case, the value keeps the same as is stated above. As $\mathrm{S}$ increases, the value rapidly decreases. As $m$ increases, the value increases slightly.

$Z$ can be used as an absolute deterioration factor for machine diagnosis. We name this "Absolute Deterioration Factor of Second Order Correlation". Varying $N$, the number of data, its characteristics is examined. When $N$ is smaller, sensitivity is better.

Kurtosis has a value of 3.0 under normal condition and the value generally goes up as the deterioration proceeds. This function is an absolute index of deterioration factor as Bicoherence which has a value from 1 to 0 .

These results show that the newly presented one is very sensitive compared with the results obtained so far. This calculation method is simple enough to be executed even on a pocketsize calculator and is very practical at the factory of maintenance site. 


\section{Remarks}

Here, we introduced an absolute deterioration factor of second order correlation. Varying $N$, the number of data, its characteristics is examined. When $N$ is smaller, sensitivity is better. Therefore this parameter can be used as an index for the machine diagnosis of early failure stage especially.

The steps for the failure detection by this method are as follows.

1. Prepare a standard $Z$ Table for each normal or abnormal level

2. Measure peak values by signal data and compare the peak ratio to the normal data

3. Calculate $Z$ by (34)

4. Judge the failure level by the score of $Z$

Preparing standard Table of $Z$ for each normal and abnormal level, we can easily judge the failure level only by taking ratio of peak value to the normal level and calculating $Z$ by (34). This method is simple enough to be carried out even on a pocketsize calculator and is very practical at the factory of maintenance site. This can be installed in microcomputer chips and utilized as the tool for early stage detection of failure.

Here, reviewing the characteristic of $Z$, it is a $\bar{\rho}_{1}^{2}$ itself. Absolute deterioration factor using second order autocorrelation function is thus proved to be a simple function and has a good characteristic for failure detection.

\section{Conclusions}

Simplified calculation method of coefficient of autocorrelation function of $1^{\text {st }}$ order lag was introduced and the new absolute deterioration factor of correlation of four variables was introduced consecutively. It was examined that it was a sensitive good parameter for machine diagnosis. We named this as "Absolute Deterioration Factor of Second Order Correlation". Three cases in which the rolling elements number was nine, twelve and sixteen were examined and compared. Generally, when $\mathrm{N}$ grows large, the value decreases slightly except for the singular case. Under the singular case, the value keeps the same as is stated above. As $\mathrm{S}$ increases, the value rapidly decreases. As $m$ increases, the value increases slightly. Varying $N$, the number of data, its characteristics were examined. When $N$ was smaller, sensitivity was better. Therefore, judging from these results, our method was properly considered that this parameter could be used as an index for the machine diagnosis of early failure stage especially. This calculation method was simple enough to be executed even on a pocketsize calculator and was very practical at the factory or maintenance site. This could be installed in microcomputer chips and utilized as the tool for early stage detection of failure. Although it has a limitation that it is restricted in the number of research, we could obtain the fruitful results. To confirm the findings by utilizing the new consecutive visiting records would be the future works to be investigated

The effectiveness of method should be examined in various cases.

\section{References}

Bolleter, U. (1988). Blade Passage Tones of Centrifugal Pumps. Vibration, 4(3), 8-13.

Maekawa, K., S. Nakajima, \& T. Toyoda (1997). New Severity Index for Failures of Machine Elements by Impact Vibration (in Japanese). J.Sope japan, 9(3), 163-168.

Noda, B. (1987). Diagnosis Method for a Bearing (in Japanese). NSK Tec. J. (647), 33-38.

Shao, Y., K. Nezu, T.Matsuura, Y.Hasegawa, \& N. Kansawa (2001). Bearing Fault Diagnosis Using an Adaptive Filter (in Japanese). J.Sope Japan, 12(3), 71-77.

Song, J. W., H.Tin, \& T. Toyoda. (1998). Diagnosis Method for a Gear Equipment by Sequential Fuggy Neural Network (in Japanese). J. Sope Japan, 10(1), 15-20.

Takeysu, K. (1989). Watching Method of Circulation Moving Object (in Japanese). Certified Patent by Japanese Patent Angency.

Takeyasu, K., T. Amemiya, K. Ino \& S. Masuda. (2003). Machine Diagnosis Techniques by Simplified Calculation Method. IEMS, 2(1), 1-8.

Hino, M. (1977). Spectrum Analysis (in Japanese). Asakura shoten Publishing.

Tokumaru, H., T. Soeda, T. Nakamizo, \& K. Akizuki. (1982). Measurement and Calculation (in Japanese), Baifukan Publishing. 
Yamazaki, H. (1977). Failure Detection and Prediction (in Japanese). Kogyo Chosakai Publishing. Nakamizo, N. (1988). Signal Analysis and System Identification (in Japanese). Corona-sha Publishig.

Takeyasu, K., Y, Higuchi. (2006). Simplified Machine Diagnosis Techniques by Impact Vibration-Deterioration Factor of Revised Autocorrelation Function Type. Osaka Prefecture University. The Journal of Economic Studies, 51(5), 49-60. 